\title{
Factores que inciden en el rendimiento académico de los estudiantes de la Universidad Politécnica del Valle de Toluca
}

\section{Factors affecting the academic performance of students of the Universidad Politécnica del Valle de Toluca}

Elizabeth Guadalupe Chong González*

\section{RESUMEN}

Se realizó un estudio con alumnos universitarios de la UPVT, para examinar sus contextos universitario y familiar, sus percepciones acerca del apoyo que les brinda su familia, los problemas que enfrentan en su proceso académico, las expectativas propias y las familiares hacia su carrera. Los datos muestran que existe relación entre el apoyo que los estudiantes perciben y su desempeño académico, así como la importancia que tiene incluir a la familia para promover y elevar el rendimiento académico, abatir la deserción y el abandono de sus estudios. Se proponen algunas actividades para lograr la participación de la familia en el rendimiento escolar de los estudiantes a fin de coadyuvar a mejorar su desempeño escolar.

Palabras clave: rendimiento académico, educación, superior, contexto familiar, expectativas propias, estudiantes universitarios

\begin{abstract}
A study was conducted with university students in the UPVT, to examine its university and family contexts, their perceptions of support that gives your family, the problems they face in their academic process, their own expectations and family to his career. The data show that there is a relationship between perceived support students and their academic performance as well as the importance of including the family to promote and raise the academic performance and fold desertion and abandonment of their studies. Some activities are proposed to involve the family in the school performance of students in order to help improve their school performance.
\end{abstract}

Key words: academic performance, and higher education, family background, own expectations, university students

\section{INTRODUCCIÓN}

A lo largo del tiempo, el desempeño de los estudiantes se ha visto afectado por distintos factores, y pareciera existir un consenso de que la lista de las causas del fracaso o del éxito escolar es amplia, ya que va desde lo personal hasta lo sociocultural, la mayoría de las veces, con una mezcla de factores personales y sociales (To-

\footnotetext{
“Universidad Politécnica del Valle de Toluca; elizagu_80@yahoo.com
} 
rres y Rodríguez, 2006). En la búsqueda de impulsar un mejor desempeño académico en los estudiantes de la Universidad Politécnica del Valle de Toluca (UPVT) es importante conocer detalladamente los problemas que enfrentan, así como sus expectativas, para llevar a cabo acciones en apoyo a la mejora de su rendimiento académico y para evitar su deserción.

Los estudiantes pasan por distintos procesos de cambio en su trayectoria universitaria: la transición entre el nivel medio superior y superior, adecuarse a un nuevo sistema que los prepara para el mercado laboral, pasar de ser adolescentes a jóvenes con mayores responsabilidades, entre otros. El principal problema de los estudiantes es que, a pesar de que cuentan con mayor y mejor acceso a las tecnologías, una oferta educativa amplia y acceso a diferentes tipos de becas, esto no se traduce en una mejora sustancial de su rendimiento, lo cual trae consigo un fracaso académico. La falta de implementación de estrategias por parte los estudiantes trae como consecuencia un deterioro en el aprendizaje.

Realizar un proyecto enfocado a identificar los factores que inciden en el desempeño académico de los estudiantes de la UPVT permitirá crear mejores estrategias que impacten positivamente en su proceso de enseńanza aprendizaje, lo que se verá reflejado en una disminución de los índices de reprobación y deserción escolar. El objetivo de este proyecto es, pues, identificar y analizar los factores que inciden en el desempeño académico de los estudiantes de la UPVT, con base en los resultados obtenidos de la aplicación de un cuestionario.

\section{RENDIMIENTO ESCOLAR}

Jiménez (2000) postula que el rendimiento escolar es un nivel de conocimientos demostrado en un área o materia, comparado con la norma de edad y nivel académico, por lo que el rendimiento del alumno debería ser entendido a partir de sus procesos de evaluación; sin embargo, la simple medición o evaluación de los rendimientos alcanzados por alumnos no provee, por sí misma, todas las pautas necesarias para la acción destinada al mejoramiento de la calidad educativa. 
En el mejor de los casos, si se pretende conceptualizar el rendimiento académico a partir de su evaluación, es necesario considerar no solamente el desempeño individual del estudiante sino la manera como es influido por el grupo de pares, el aula o el propio contexto educativo.

El rendimiento escolar, incluyendo aspectos tales como el nivel de logro alcanzado en materias específicas, tasas de repetición y de retención escolar, ha sido analizado tomando en cuenta dos conjuntos de causas: aquellos aspectos relacionados con la escuela como sistema educativo, y las características que los alumnos exhiben a partir de su contexto social, de sus capacidades personales, de sus motivaciones (Mella y Ortiz, 1999).

En la explicación del rendimiento escolar, lo más importante son las características de los propios estudiantes, sus capacidades, vocación, experiencias previas, esfuerzo y disposición a aprender, sin embargo las instituciones deben ofrecer oportunidades y ambientes formativos, en términos de su calidad y pertinencia para propiciar el desempeño de los estudiantes (Aldana, et al., 2010).

Las variables que más explican el rendimiento escolar son:

- El nivel socioeconómico-cultural

- Expectativas del profesor

- Expectativas de los padres en relación con el rendimiento académico de los alumnos.

La lista de las causas del fracaso o del éxito escolar es amplia, ya que va de lo personal a lo sociocultural, la mayoría de las veces, con una mezcla de factores personales y sociales.

\section{Impacto de las condiciones socioeconómicas en el rendimiento escolar}

Las condiciones socioculturales materiales que intervienen en el desempeńo y aprendizaje escolar, impelen a indagar en la capacidad de las familias, para apoyar con recursos materiales y culturales al estudiante.

Así quizá una mayor disponibilidad del ingreso a nivel familiar puede impactar decisivamente en el resultado del alumno, 
porque implicaría una capacidad mayor de pagar una escuela mejor, una infraestructura tal que permitiría condiciones favorables para el estudio, un mejor transporte, entre otras.

Piñeiro y Rodríguez (1998) mencionan que el nivel socioeconómico del estudiante tiene efectos positivos sobre el rendimiento académico del mismo. Lo cual subraya la importancia de la responsabilidad compartida entre la familia, la comunidad y la escuela en el proceso educativo.

\section{Contexto familiar}

Un buen aprendizaje y un buen rendimiento académico dependen, en gran medida, de la dedicación que el estudiante preste a las tareas escolares durante el tiempo que está en su hogar, aunque, al contrario de lo que pudiera pensarse, no es tan importante el tiempo que se invierte en el estudio como la calidad de este (Torres y Rodríguez, 2006).

Es sustancial tomar conciencia de que estudiar es un trabajo que requiere esfuerzo y sacrificio, tanto por parte de los padres como de los hijos. De este modo, la influencia paterna es importante en cuanto a los aspectos ambientales y la motivación, en tanto que del estudiante depende la organización del tiempo, la elección de la técnica de estudio, el compromiso y la motivación.

La situación económica de la familia repercute en el estudiante cuando, al no ser cubiertas las necesidades primarias, la educación o las tareas escolares son devaluadas y se da prioridad al trabajo remunerado; por lo tanto, la familia exige al estudiante que ayude económicamente para resolver dichas necesidades. Así mismo, hay estudiantes con hijos, por lo que sus obligaciones prioritarias son otras, o estudiantes que, aunque no trabajan fuera de la casa, se encargan de todas las labores domésticas, y en ocasiones de la crianza de sus hermanos más pequeños.

Los padres son el agente socializador fundamental y, desde el comienzo de la vida, se comunican con los alumnos, transmitiéndoles su nivel cultural por medio del lenguaje y la relación afectiva. En la mayor parte de las investigaciones efectuadas al respecto, el nivel de educación formal de las madres tiene una 
enorme potencialidad explicativa en el desarrollo del estudiante (Mella y Ortiz, 1999).

\section{Contexto escolar}

En su búsqueda por mejorar la calidad de la educación y con la finalidad de responder a su compromiso social, las escuelas han prestado atención al quehacer académico de sus estudiantes; dentro de algunas instituciones de educación superior se están llevando a cabo diversas acciones para apoyar la mejora del rendimiento académico para evitar la deserción de los estudiantes (Aldana, et al., 2010).

Torres y Rodríguez (2006) han detectado tres periodos críticos en la trayectoria escolar universitaria:

- En la transición entre el nivel medio superior y la licenciatura.

- Durante el proceso de admisión.

- Cuando el estudiante no logra un adecuado rendimiento académico en las asignaturas del plan de estudios y la institución no le proporciona las herramientas necesarias para superar las deficiencias académicas.

Una acción que están llevando a cabo diversas instituciones es apoyar al alumno por medio de la tutoría; de esta forma, el profesor podrá alcanzar una mejor comprensión de los problemas que enfrenta el alumno en su adaptación al ambiente universitario, de las condiciones individuales para un desempeño aceptable durante su formación y del logro de los objetivos académicos que le permitirán enfrentar los compromisos de su futura práctica profesional (Torres y Rodríguez, 2006).

De tal suerte, se procura, por medio de la tutoría:

- Apoyar al alumno en el desarrollo de una metodología de estudio y trabajo apropiado para las exigencias del primer ańo de la carrera.

- Ofrecerle apoyos y supervisión en temas de mayor dificultad en las diversas asignaturas y crear un clima de confianza que permita conocer aspectos de su vida personal que influyen directa e indirectamente en su desempeño. 
- Señalar y sugerir actividades extracurriculares para favorecer su desarrollo profesional integral y,

- Brindarle información académico-administrativa, según sus peticiones o necesidades.

Otro factor importante en el rendimiento escolar del alumno es la motivación, la cual es un proceso general por el cual se inicia y se dirige una conducta hacia el logro de una meta. Así que, motivar a los estudiantes, implica fomentar sus recursos internos, su sentido de competencia, autoestima, autonomía y realización (Edel, 2003).

\section{MÉTODO}

Se realizó investigación bibliográfica y hemerográfica para elaborar el marco teórico. Además de la información estadística obtenida de diferentes organismos, se efectuó levantamiento de información mediante la aplicación de un cuestionario (ver anexo) que permita conocer los factores que inciden en el desempeño académico del estudiante de la UPVT.

Para la aplicación del instrumento se tomó una muestra de todos los estudiantes de la UPVT, de las distintas carreras que se imparten (ver cuadro 1). El levantamiento de la información se llevó a cabo en los meses de octubre y noviembre de 2014.

\section{Resultados}

Los resultados se analizan en tres secciones: datos demográficos, rendimiento escolar y contexto familiar.

\section{Datos demográficos}

La edad promedio de los alumnos que participaron en esta muestra fue de 20 años, con $67 \%$ de hombres y 33\% de mujeres (ver gráfico 1), está situación se da porque la Universidad cuenta con carreras de ingeniería, donde principalmente se ubican los varones. 
FACTORES QUE INCIDEN EN EL RENDIMIENTO ACADÉMICO DE LOS ESTUDIANTES DE LA UPVT

CUADR0 1. Tamaño de la muestra de los estudiantes de la UPVT

\begin{tabular}{|l|c|c|}
\hline \multicolumn{1}{|c|}{ Carrera } & Número de estudiantes & Tamaño de la muestra \\
\hline Ingeniería Industrial & 837 & 78 \\
\hline Ingeniería Mecatrónica & 642 & 60 \\
\hline Ingeniería en informática & 530 & 49 \\
\hline Ingeniería en Biotecnología & 604 & 56 \\
\hline $\begin{array}{l}\text { Licenciatura en Negocios } \\
\text { Internacionales }\end{array}$ & 602 & 56 \\
\hline Ingeniería en Mecánica Automotriz & 436 & 41 \\
\hline Ingeniería en Energía & 90 & 8 \\
\hline Total de alumnos & 3741 & 348 \\
\hline
\end{tabular}

\section{GRÁFICO 1. Distribución de los estudiantes de la UPVT, por sexo}

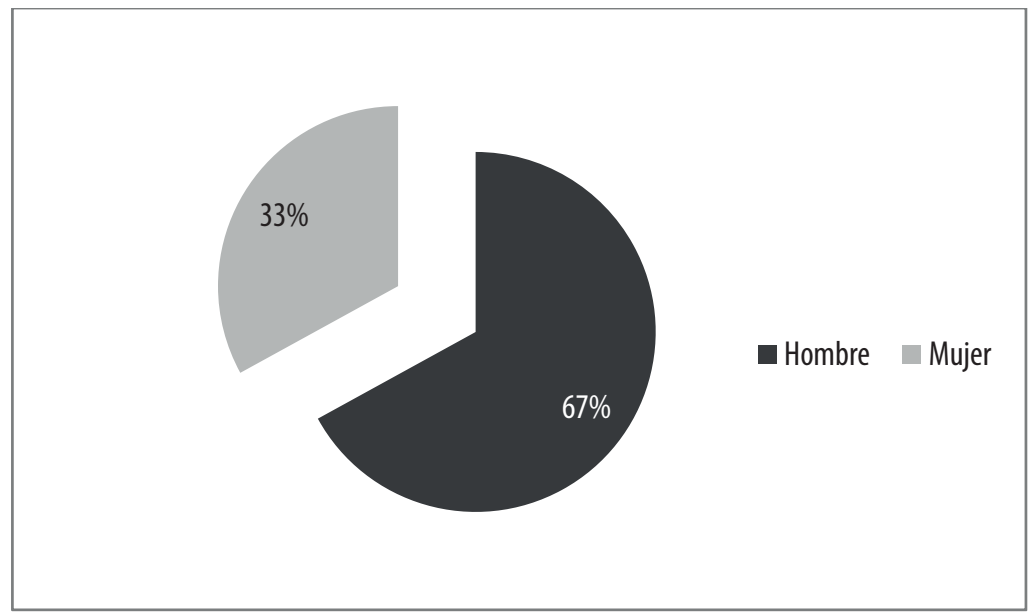

De los 348 encuestados, el $79 \%$ pertenece a una familia nuclear (padre, madre e hijos), 15\% con madre o Padre y/o hermanos, $5 \%$ vive solo y $1 \%$ pertenece a una familia extensa. Los porcentajes del tipo de familia a la que pertenecen concuerda con la estructura familiar propia del país, donde la mayoría pertenece a una familia nuclear $y$, en menor medida, a familias uniparentales o extensas. 
$87 \%$ de los participantes mencionó pertenecer a la clase media, mientras que $12 \%$ a la clase baja y $1 \%$ a la clase alta. $83 \%$ usa trasporte público para llegar a la escuela y $17 \%$ usa su propio vehículo. La mayoría no trabaja $(71 \%)$ y principalmente provienen del municipio de Toluca y Zinacantepec. El tiempo que les toma trasladarse de su casa a la UPVT se muestra en el siguiente gráfico, lo que implica que les consume más de una hora en promedio esa actividad.

GRÁFICO 2. Tiempo que tarda el alumno en llegar de su casa a la UPVT

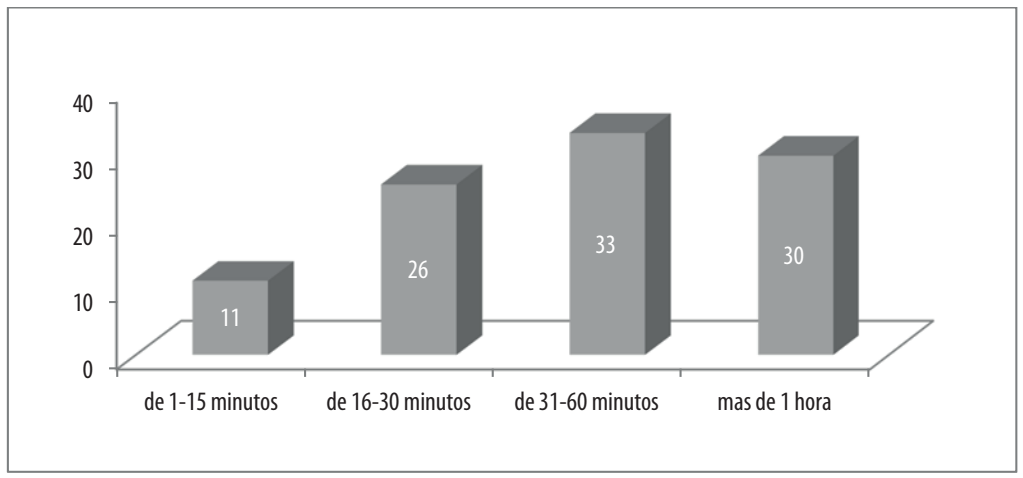

\section{Rendimiento Escolar}

En cuanto a los factores que consideraron propiciaban su aprovechamiento, estos fueron la preparación de los profesores (45\%), sus ganas de superarse (39\%), las actividades extracurriculares (11\%) y mantener relaciones familiares sanas (5\%) (ver gráfico 3). Entre los obstáculos que hallaron para su aprovechamiento académico, mencionaron la falta de tiempo $(31 \%)$, la poca preparación de algunos profesores (31\%), el trabajo (16\%), actividades extracurriculares $(13 \%)$ y las relaciones familiares $(8 \%)$. 
GRÁFICO 3. Factores que contribuyen al aprendizaje del alumno

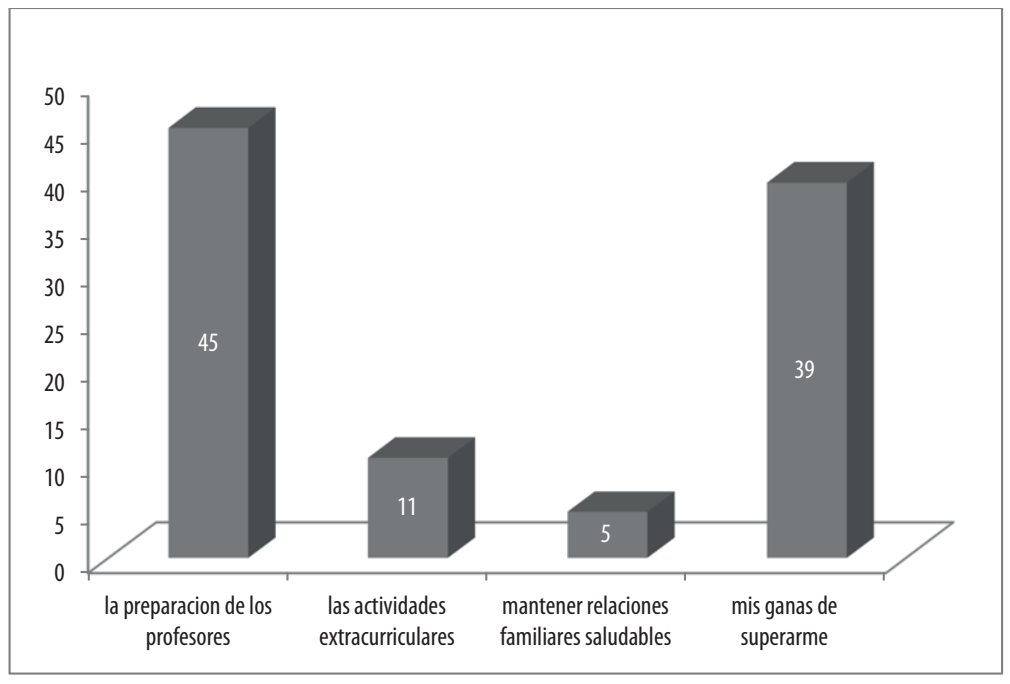

La mayor parte de los encuestados creían poder mejorar su aprovechamiento académico si contaran con el material adecuado para estudiar; aunque manifestaron no acudir cotidianamente a la biblioteca, les gustaría tener más recursos económicos para comprar sus propios libros y tenerlos en casa. Otra de las actividades que juzgaron importante para lograr un óptimo aprovechamiento se relaciona con su estado anímico y su motivación e interés de estudiar, con tener tiempo y un lugar adecuado, para ello $50 \%$ dijo hacer la tarea en su habitación, el $18 \%$ en la salacomedor, otro $18 \%$ no tiene un lugar en específico, el 13\% en el estudio y el $1 \%$ en el trabajo. Este factor involucra diferentes problemas o contratiempos que enfrentan con la familia, maestros, autoridades escolares, amigos, así como problemas sentimentales o emocionales. Esta etapa de desarrollo del estudiante universitario es compleja y provoca diversos ajustes y desajustes en su vida cotidiana, plan de vida, emociones y perspectivas.

Otro factor importante que influye en el rendimiento académico del estudiante es qué tan motivado se encuentra en relación a la carrera que estudia, el gráfico 4 muestra que el 38\% eligió su carrera porque realmente le gusta. 


\section{GRÁFICO 4. Motivo de elección de la carrera}

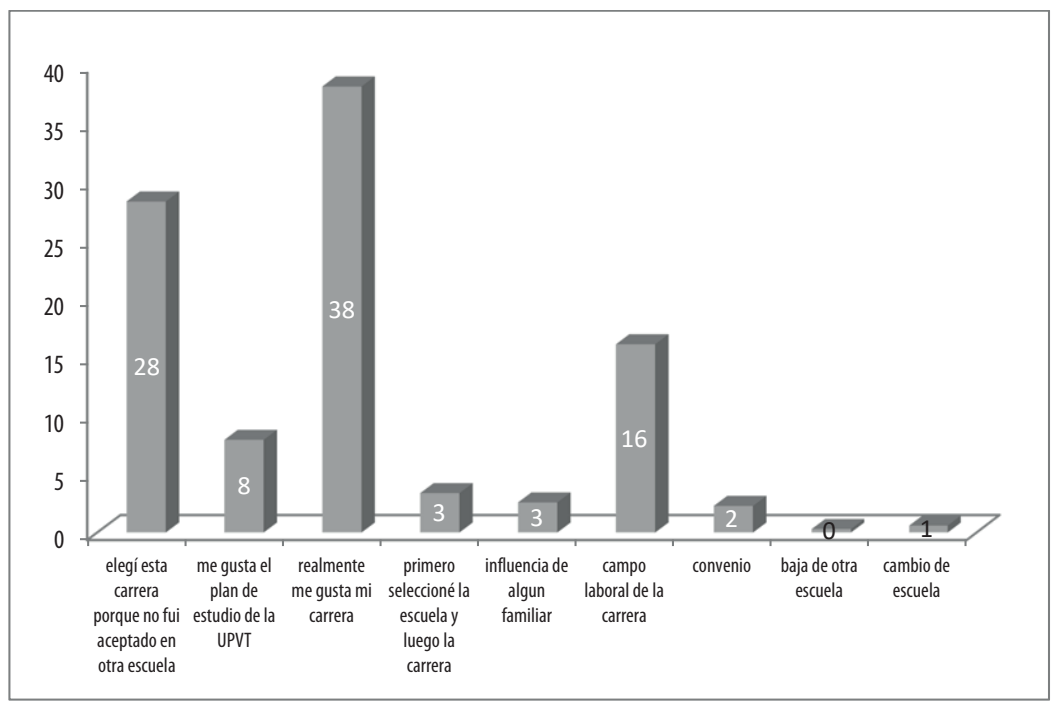

En el gráfico anterior causa preocupación el 28\% que se encuentra en la UPVT porque no fue aceptado en otra institución, lo que debe impulsar a la UPVT a generar estrategias para motivar y orientar al alumno en su proceso de adaptación en la escuela que se encuentra, a pesar de que no fue ésta su primera opción (ver gráfico 5).

\section{GRÁFICO 5. La carrera que estudian fue su primera opción}

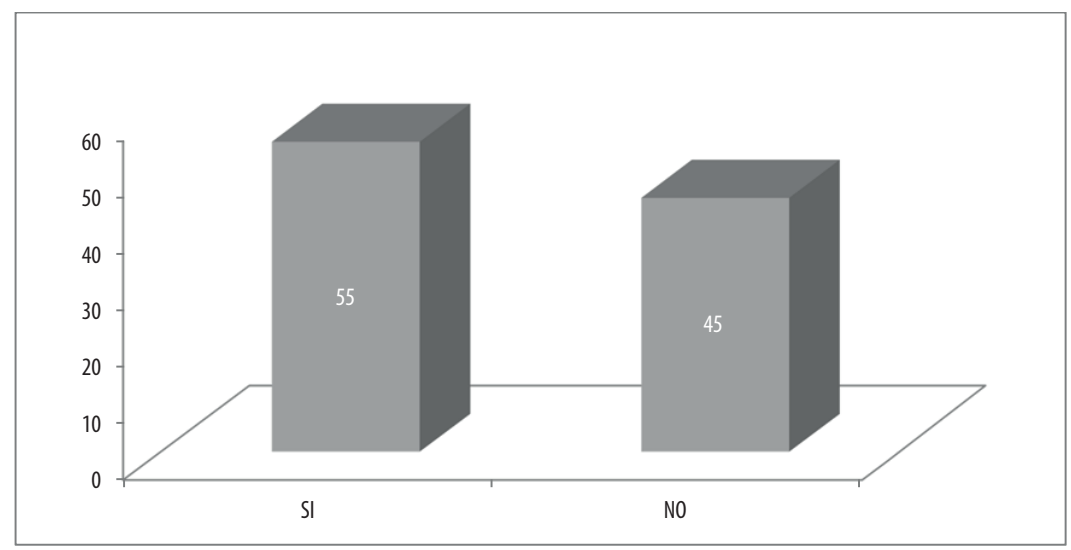




\section{Contexto Familiar}

El 61\% afirmó que sus relaciones familiares eran buenas, 28\% excelentes, $10 \%$ regulares y $1 \%$ malas. De los alumnos encuestados, el 95\% percibe que su familia los apoya en sus estudios. En general señalaron que su familia respeta sus tiempos de estudio. Efectivamente, los resultados muestran que son muy similares las expectativas del estudiante con las expectativas que tiene su familia respecto a sus estudios (ver gráfico 6 y 7). Esta situación los impulsa a tener mejores perspectivas de vida, lo que se ve reflejado en un mejor rendimiento escolar, debido a que están respaldados por sus familias ya que aprueban y apoyan a sus hijos en sus estudios.

GRÁFICO 6. Expectativas del alumno al terminar su carrera

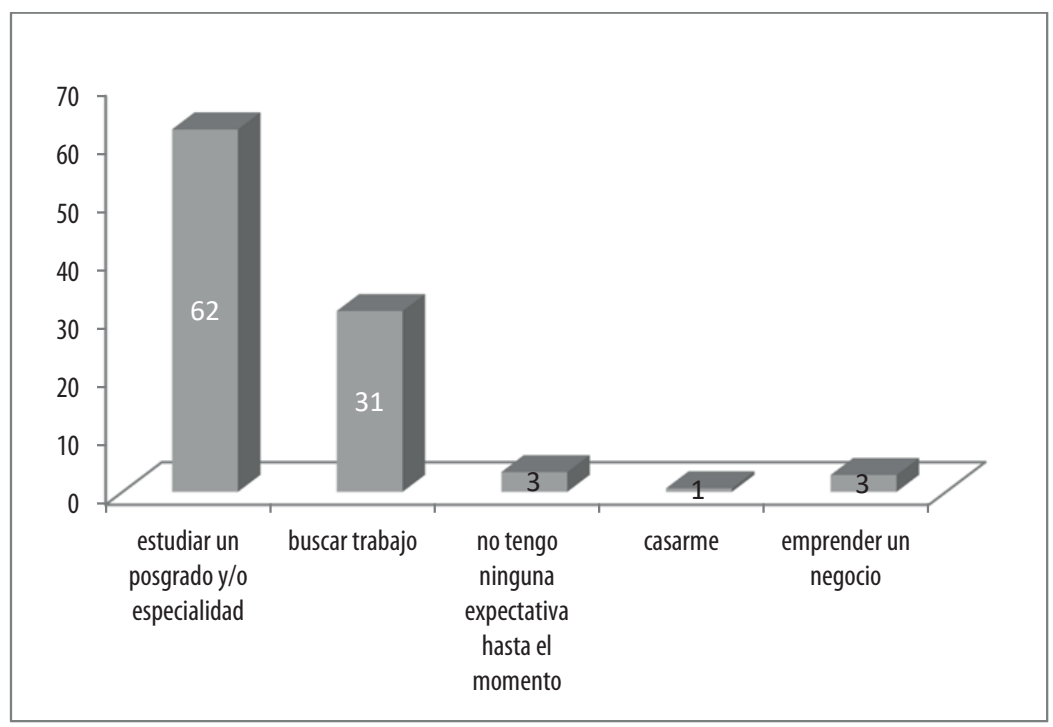


REVISTA LATINOAMERICANA DE ESTUDIOS EDUCATIVOS, VOL. XLVII, NÚM. 1, 2017

GRÁFICO 7. Expectativas familiares al terminar su carrera

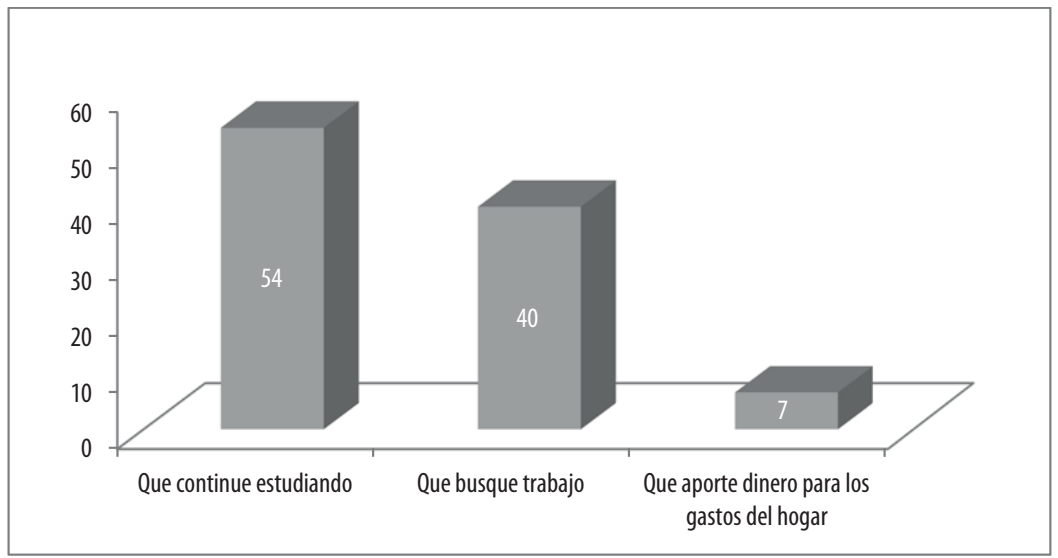

El $24 \%$ tienen un promedio de 8 , lo que se califica como un buen promedio en la carrera universitaria. Es importante recordar lo anterior porque es posible asociarlo con la percepción que tienen los alumnos de su contexto familiar, ya que la mayoría lo considera positivo (ver gráfico 8).

GRÁFICO 8. Influye tu familia en tu aprendizaje

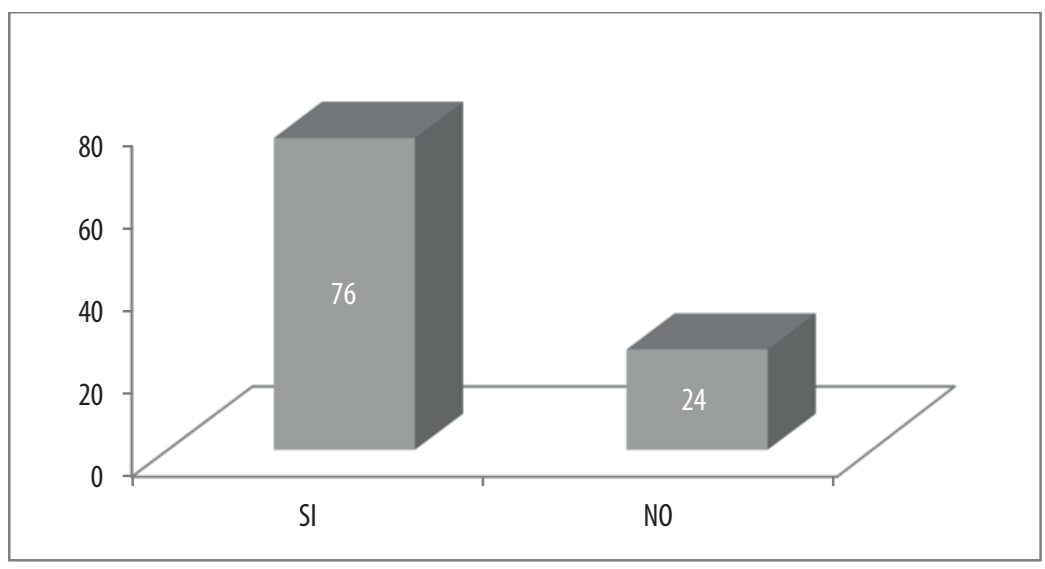


Lo anterior implica que no solamente en niveles escolares más abajo, existe una relación positiva entre el contexto familiar y el escolar, sino que aun en el nivel superior tales contextos se afectan directamente.

\section{CONCLUSIÓN}

En este estudio se muestra que los estudiantes de la UPVT consideran que los factores que contribuyen a su aprendizaje son la preparación de los profesores, sus ganas de superarse y, en menor medida, sus relaciones familiares; es importante reflexionar sobre el papel que juega la escuela en este proceso, debido a que debe de proveer al estudiante de todas las herramientas necesarias, para que este pueda mejorar su rendimiento académico.

Las instituciones de educación superior están llevando a cabo diversas acciones para apoyar la mejora del rendimiento académico y evitar la deserción de los estudiantes. Para lograr la transformación del servicio que se ofrece a los estudiantes de nivel superior se ha propuesto la tutoría como un acompañamiento y apoyo docente de carácter individual. Por medio de la tutoría el profesor puede alcanzar una mejor comprensión de los problemas que enfrenta el alumno en su adaptación al ambiente universitario, de las condiciones individuales para un desempeño aceptable durante su formación, y del logro de los objetivos académicos que le permitirán enfrentar los compromisos de su futura práctica profesional.

Todas estas acciones son importantes y transformadoras en la educación superior ya que permiten mejorar el desempeño y la eficiencia en las licenciaturas, así como incrementar la retención, las tasas de egreso y la titulación. Sin embargo, aún no se ha considerado relevante incluir la exploración y estudio de las familias de los estudiantes universitarios, sería conveniente incluir el contexto familiar como promotor del desarrollo académico de los alumnos, debido que existe una relación positiva entre las expectativas de los estudiantes y las expectativas de su familia en relación a sus estudios.

La manera en que puede lograrse que el contexto familiar contribuya al éxito escolar es quizá mediante cursos o talleres para 
los alumnos y los padres de familia, en los que se aborden temas que beneficien la relación familiar, la comunicación, la solución de problemas y demás, a fin de que los estudiantes puedan establecer una relación eficaz con su familia, y gracias a los cuales la familia conozca mejor lo que los alumnos estudian, la importancia de que cuenten con un lugar y tiempo para su actividad, los problemas a los que se enfrentan, sus contraindicaciones, avances y triunfos, el campo de trabajo futuro y otros.

Es importante que se establezcan vínculos entre las universidades y las familias de los estudiantes para así coadyuvar al beneficio de la actividad educativa de los alumnos universitarios y contribuir a frenar el problema más grave del sistema educativo mexicano: la deserción.

El generar proyectos cuya finalidad sea contribuir al mejor desempeño de los estudiantes y mejor funcionalidad de las escuelas de educación superior, traerá consigo la incorporación de mejores personas al ámbito laboral y social, lo que se verá traducido en un desarrollo social, económico y cultural del país.

\section{REFERENCIAS BIBLIOGRÁFICAS}

Aldana, Kelsy, Reyna Pérez de Roberti y Ayolaida Rodríguez Miranda, "Visión del desempeño académico estudiantil en la Universidad Centroccidental Lisandro Alvarado", en Revista Compendium, vol. 13, núm. 24, Universidad Centroccidental Lisandro Alvarado, Venezuela, 2010, pp. 5-21.

Edel, Rubén, "El rendimiento académico: concepto, investigación y desarrollo", en Revista electrónica de iberoamericana sobre calidad, eficacia y cambio en educación, REICE, vol.1, núm. 2, Madrid, España, 2003, pp.1-15.

Jiménez, María Isabel y López-Zafra, Esther, "Inteligencia emocional y rendimiento escolar: estado actual de la cuestión", en Revista Latinoamericana de Psicología, vol. 41, núm.1, Fundación Universitaria Konrad Lorenz, Colombia, 2009, pp. 69-79.

Jiménez, María Isabel, "Competencia social: intervención preventiva en la escuela”, en Infancia y Sociedad, núm. 24, 2000, pp. 21-48. 
Mella, Orlando e Iván Ortiz. "Rendimiento escolar. Influencias diferenciales de factores externos e internos", en Revista Latinoamericana de Estudios Educativos, vol. 29, núm.1, Centro de Estudios Educativos, A. C. México, 1999, pp. 69-92.

Peñaloza, Eduardo, Estrategias docentes con tecnologías: guía práctica, México, Pearson, 2013.

Pimienta, Julio Herminio, Estrategias de enseñanza aprendizaje, México, Pearson, 2012.

Piñeiro, Luis Jaime y Alberto Rodríguez, "Los insumos escolares en la educación secundaria y su efecto sobre el rendimiento académico de los estudiantes", Human Development Department, LCSHD, Paper Series, núm. 36, The World Bank, Latin America the Caribbean regional Office, 1998.

Torres, Laura y Norma Rodríguez, "Rendimiento académico y contexto familiar en estudiantes universitarios", en Revista Enseñanza e investigación en psicología, vol.11, núm.2, Universidad Veracruzana, Xalapa, México, 2006, pp. 255-270. 


\section{ANEXO}

CUESTIONARIO No.

Objetivo: Conocer los factores socioeconómicos que afectan el desempeńo académico de los estudiantes de la Universidad Politécnica del Valle de Toluca.

Instrucciones: Lee cuidadosamente las siguientes preguntas y selecciona aquella que te parezca adecuada. Utiliza el apartado "otro" para escribir una respuesta que no se encuentre entre las diferentes opciones.

\section{Factores sociodemográficos}

\begin{tabular}{|l|l|l|}
\hline 1. Edad: & 2. Sexo:M() F() & 3. Municipio donde vives: \\
\hline $\begin{array}{l}\text { 4. Carrera: } \\
\text { Cuatrimestre: }\end{array}$ & & 5. Promedio general actual: \\
\hline
\end{tabular}

\section{Factores sociales}

6. ¿A qué clase social consideras que perteneces?

$$
\text { Alta ( ) Media ( ) Baja ( ) }
$$

7. ¿Actualmente trabajas?
a) Sí
b) No

8. ¿Cuántos dependientes económicos tienes?

Ninguno ( ) Esposa ( ) Hijos ( ) Ambos ( ) Otro ( )

9. ¿Cuánto tiempo haces de tu casa a la UPVT?

\begin{tabular}{|l|l|l|l|}
\hline a) De 1-15 minutos & b) De 16-30 minutos & c) De 31-60 minutos & d) Más de 1 hora \\
\hline
\end{tabular}

10. ¿Cuál es tu medio de transporte a la UPVT?

\begin{tabular}{|l|l|l|}
\hline a) Transporte público & b) Automóvil propio & c) Otro \\
\hline
\end{tabular}

11. ¿La distancia entre el lugar en donde vives y UPVT, influyó en tu decisión al seleccionar la escuela?
a) Sí
b) No

\section{Factores escolares}

12. ¿Tienes beca? No ( ) Síl ( ) ¿Quién te la otorga?

13. ¿Recibiste orientación vocacional antes de elegir tu carrera?
a) Sí
b) No

14. La carrera que estudias, ¿fue tu primera opción?
a) Sí
b) No 
15. Subraya la opción que te llevó a elegir la carrera que estudias:

a) Elegí esta carrera porque no fui aceptado en otra escuela y entre la oferta académica de UPVT busqué la que se parece a la que carrera que quería estudiar.

b) Me gusta el plan de estudios de UPVT

c) Realmente me gusta mi carrera

d) Primero seleccioné la escuela y después una carrera que me llamara la atención

e) Influencia de algún familiar

f) Campo laboral de la carrera

g) Otro

16. En general, ¿tus maestros te parecen preparados y/o adecuados para la materia que les asignan?
a) Todos
b) Casi todos
c) Casi ninguno
d) Ninguno

17. ¿Cómo calificarías el nivel de enseñanza de UPVT?
a) Pésimo
b) Malo
c) Regular
d) Bueno
d) Excelente

\section{Factores familiares}

18. ¿Con quién vives?
a) Ambos padres
b) Madre
c) Padre
d) Conyugue
e) Solo
f) Otro

19. ¿Te apoya tu familia en tus estudios?
a) Sí
b) $\mathrm{No}$

20. Además de estudiar, ¿qué obligaciones tienes en tu casa?

a) Ninguna b) Limpieza del hogar c) Cuidar a tus hermanos y/o algún otro miembro de la familia d) Aportar dinero para los gastos del hogar

e) Otro

21. ¿En qué lugar especifico haces la tarea?

a) En mi habitación b) Cuarto de estudio c) Sala-Comedor d) No tengo un lugar en específico e) Otro

22. Las relaciones familiares en tu casa son, en general:

\begin{tabular}{l|l|l|l|l} 
a) Excelentes & b) Buenas & c) Regulares & d) Malas
\end{tabular}

23. ¿Qué opina tu familia de que estés estudiando?

a) Es bueno que me supere b) No tiene importancia que lo haga

c) Debería abandonar la escuela y buscar trabajo d) No opinan nada al respecto e) Otro 
24. ¿Te exige tu familia que saques buenas calificaciones?
a) Sí
b) $\mathrm{No}$

25. ¿Qué expectativas tiene tu familia para cuando termines la carrera?

a) Que continúe estudiando b) Que busque trabajo c) Aporte dinero para los gastos del hogar d) Otro

26. ¿Crees que tu familia influye en tu aprendizaje?
a) Sí
b) No

27. Marca con una X el nivel de estudios alcanzado por sus padres

\begin{tabular}{|l|l|l|l|l|l|l|l|}
\hline Madre & Ninguno & Primaria & Secundaria & Bachillerato & Licenciatura & Especialidad & Posgrado \\
\hline Padre & Ninguno & Primaria & Secundaria & Bachillerato & Licenciatura & Especialidad & Posgrado \\
\hline
\end{tabular}

\section{Factores personales}

28. ¿Qué factores crees que contribuyen a tu aprendizaje?
a) La preparación de los profesores
b) Las actividades extracurriculares
c) Mantener relaciones familiares saludables
d) Mis ganas de superarme
e) Otro

29. ¿Cuáles crees que lo obstaculizan?
a) La preparación de los profesores
b) Las actividades extracurriculares
c) Relaciones Familiares
d) Falta de tiempo
e) El trabajo y/o alguna actividad extra
f) Otro

30. ¿Cuántas horas en promedio dedicas a hacer la tarea cada día?

\begin{tabular}{ll|l|l|l} 
a) Menos de una hora & b) 1-2 horas & c) 3-5 horas & d) Más de 5 horas
\end{tabular}

31. ¿Qué expectativas tienes para cuando termines tu carrera?

a) Estudiar un posgrado y/o especialidad

b) Buscar trabajo

c) No tengo ninguna expectativa hasta el momento

d) Casarme

e) Otro 